\title{
Hybrid Control Strategies in ABS
}

\author{
Tor A. Johansen ${ }^{2,3}$, Jens Kalkkuhl ${ }^{1}$, Jens Lüdemann ${ }^{1}$ and Idar Petersen ${ }^{2}$ \\ ${ }^{1}$ DaimlerChrysler AG, Research and Technology, Alt-Moabit 96A, D-10559 Berlin, Germany \\ ${ }^{2}$ SINTEF Electronics and Cybernetics, Trondheim, Norway \\ ${ }^{3}$ Dept. Engr. Cybernetics, Norwegian Univ. Sci. Tech., N-7491 Trondheim, Norway
}

\begin{abstract}
The ABS control problem is described, with a discussion on relevant hybrid control aspects. Next, we comment on conventional ABS design methods and present some new ideas and results on model-based ABS control design that relies on elements of hybrid control.

\section{Introduction}

Anti-lock brake systems (ABS) control the brake torque of each wheel to prevent it from getting locked in the process of braking. This is important to maintain maneuverability of the vehicle. Recent braking systems aim to control the slip of the wheel to arbitrary setpoints given by higher level control systems such as the electronic stability program (ESP) for anti-skid. Current production ABS systems are highly sophisticated hybrid controllers. Initially the hybrid nature of the controller arose from the requirement to use cheap and simple hydraulic valves with three-point-characteristics as actuators. Furthermore, the hybrid nature of these controllers facilitates an adaptive behaviour with respect to the highly uncertain tyre characteristics and the fast changing road surface properties. ${ }^{1} 2$
\end{abstract}

\section{Equations of motion of a quarter car}

The problem of ABS control is best explained by looking at a quarter car model [1]. The model consists of a single wheel attached to a mass $m$. While the wheel moves driven by inertia of the mass $m$ in the direction of the velocity $v$, a tyre reaction force $F_{x}$ is generated by the friction between the tyre surface and the road surface. The tyre reaction force will generate a torque that initiates a rolling motion of the wheel causing an angular velocity $\omega$. A brake torque applied to the wheel will act against the spinning of the wheel causing a negative angular acceleration. The equations of motion of the quarter car are

$$
\begin{aligned}
m \dot{v} & =-F_{x} \\
J \dot{\omega} & =r F_{x}-T_{b} \operatorname{sign}(\omega)
\end{aligned}
$$

where

$\begin{array}{ll}v & \text { longitudinal speed at which the car travels } \\ \omega & \text { angular speed of the wheel } \\ F_{z} & \text { vertical force } \\ F_{x} & \text { tyre friction force } \\ T_{b} & \text { brake torque } \\ r & \text { wheel radius } \\ J & \text { wheel inertia }\end{array}$

The tyre friction force is given by $F_{x}=F_{z} \cdot \mu\left(\lambda, \mu_{H}, \alpha\right)$ and the friction coefficient $\mu$ is a nonlinear function of

\footnotetext{
${ }^{1}$ This work was sponsored by the European Commission under the ESPRIT Long Term Research project $28104 \mathcal{H}^{2} \mathcal{C}$.

${ }^{2}$ Corresponding author: Tor.Arne.Johansen@itk.ntnu.no.
}

$\begin{array}{ll}\lambda & \text { tyre slip } \\ \mu_{H} & \text { friction coefficient between tyre and road } \\ \alpha & \text { slip angle of the wheel }\end{array}$

The slip $\lambda=(v-\omega r) / v$ describes the normalised difference between horizontal speed $v$ and speed of the wheel perimeter $\omega r$. The slip value of $\lambda=0$ characterises the free motion of the wheel where no friction force $F_{x}$ is exerted. If the slip attains the value $\lambda=1$ then the wheel is locked which means that it has come to a standstill. The friction coefficient $\mu_{H}$ and the shape of the friction curve $\mu(\cdot)$ can vary in a very wide range, depending on the road conditions (dry, wet) and surface (ice, snow, gravel, asphalt etc.), slip angle of the wheel (due to steering), and type of tyre (summer, winter). To analyse the dynamics of system (1)-(2) a change of variables is carried out where the angular speed of the wheel $\omega$ is replaced by the slip $\lambda$ (assuming $\omega \geq 0$ and $v>0)$ :

$$
\begin{aligned}
\dot{\lambda} & =-\frac{1}{v}\left\{\frac{1}{m}(1-\lambda)+\frac{r^{2}}{J}\right\} F_{z} \mu\left(\lambda, \mu_{H}, \alpha\right)+\frac{1}{v} \cdot \frac{r}{J} T_{b} \\
\dot{v} & =-\frac{1}{m} F_{z} \mu\left(\lambda, \mu_{H}, \alpha\right)
\end{aligned}
$$

It can be seen that the time scale of the slip dynamics (3) scales with speed $v$. The qualitative dynamic behaviour of slip is not affected by speed.

\section{Control strategy - conventional ABS}

The basic control-philosophy of conventional ABS systems is a combination of slip control and wheel acceleration control $[1,2,3]$. The control objective is to keep wheel slip at a specified set point or to maintain a specified wheel acceleration. Slip control works satisfactorily for non-decreasing tyre force characteristics while wheel acceleration control tends to work better for tyre characteristics that have a pronounced maximum. In conventional ABS systems the actuators are hydraulic solenoid valves which have three modes, i) brake pressure increase, ii) brake pressure hold, and iii) brake pressure reduction. The controller is switched on when the acceleration of the wheel drops below a certain value for a sufficient period of time. As long as the ABS is active the switching between the different actuator modes is controlled either via several slip and acceleration thresholds or by defining a switching surface using a weighted sum of slip and acceleration. In practice ABS controllers have been shown to be highly adaptive since they can tolerate a considerable amount of uncertainty in the tyre force characteristics and the friction coefficient. Any production ABS incorporates a number of subsystems apart from the wheel slip controller. Among these systems the logic responsible for coordinating the four wheel slip controllers is of particular importance. The wheel slip controllers for each wheel are (as safety devices) only active in critically situations. Thus, each controller is switched off and the brake is set to manual operation when the wheel is no longer in danger of being locked. On the other hand the slip controller has 


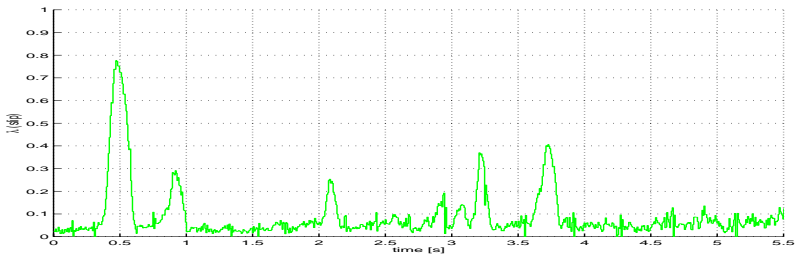

Figure 1: Conventional production ABS on snow.

to be switched on early enough to prevent the wheel from locking. The corresponding switching logic is crucial for the functionality of the ABS and constitutes an inherently hybrid control design task.

\section{Control strategy - model-based ABS}

There is an increasing interest in automotive brake-bywire technologies. Such technologies will require new types of brake actuators such as electro-mechanical or electrohydraulic brakes. A main feature of electro-mechanical and electro-hydraulic brakes is that they allow adjustment of the brake force more accurately than conventional brakes with solenoid valves. A major issue of future automotive control is that the ABS will interact with other systems such as cruise control and anti-skid-control. We are currently studying how modern hybrid control approaches can contribute to a more systematic, model based ABS design.

\subsection{Explicit LQR with constraints}

There has recently been developed algorithms for efficient real-time implementation of constrained LQ controllers (socalled explicit methods that does not rely on real-time optimization but rather on explicit evaluation of the resulting piecewise linear state feedback) $[4,5]$. The handling of constraints is important since for example actuator rate constraints will constitute fundamental performance limitations and are also considered to be a useful design parameter to avoid excitation of resonant suspension modes. Nonlinearities such as the dependence on $v$ are handeled by gain-scheduling and local linearizations. The resulting controller is essentially switching between various linear control laws and therefore constitutes a hybrid controller.

\subsection{Lyapunov-based resetting adaptive nonlinear control}

The slip control problem can be viewed as a nonlinear adaptive control problem. We investigate modern adaptive design methods based on control Lyapunov functions $[6,7]$. A major challenge is to account for rapid changes in the road conditions, for example when driving on a surface that contains large patches with water or ice. In order to avoid too high adaptation gain one relies on multiple models/observers in order to detect jumps in the road/tyre friction $\mu_{H}$ and reset the parameter estimate of the adaptive algorithm accordingly. The reset algorithm is based on the control Lyapunov function with guarantees on improving the nominal performance, by introducing a hybrid/switching element in the feedback loop [8].

\section{Discussion}

The experimental results shown in Figure 1-3 are from the same test vehicle with electro-mechanical brake actuators. Note, however, that they can not be compared directly due to different road conditions and that the model-based control results are preliminary. Still, the results with the hybrid model-based approaches suggest that model-based and hybrid control has several advantages to offer in the design of ABS, both with respect to performance and effort required for development and tuning.
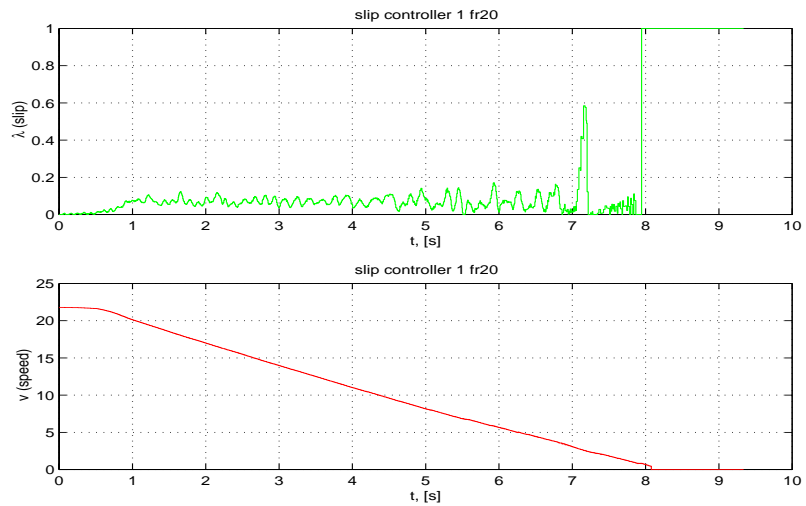

Figure 2: Experimental results: Slip with gain-scheduled explicit constrained LQ ABS brakes, on snow (setpoint is 0.07).

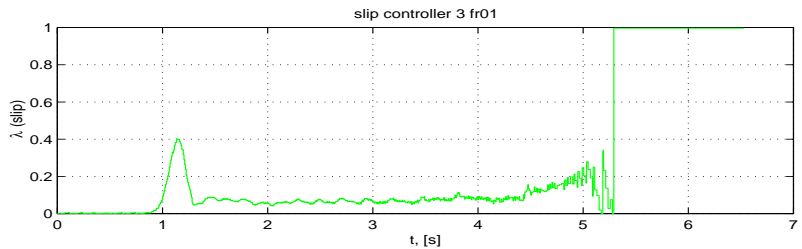

p controller 3 fro1

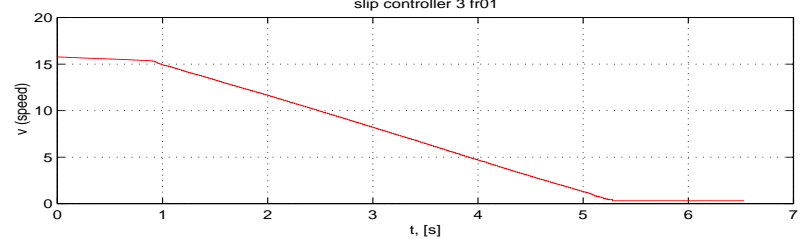

Figure 3: Experimental results: ABS brakes based on Sontag's universal controller and Lyapunov based adaptive resetting, on snow. (setpoint is $0.07)$.

\section{References}

[1] M. Burckhardt, Fahrwerktechnik: Radschlupfregelsysteme, Vogel-Verlag, Germany, 1993.

[2] P. E. Wellstead and N. Pettit, "Analysis and redesign of an antilock brake system controller", IEE Proc. D, vol. 144, pp. 413-426, 1997.

[3] Bosch GmbH (Hrsg.), Bremsanlagen für Kraftfahrzauge, Stuttgart: Robert Bosch Gmbh, 1994.

[4] A. Bemporad, M. Morari, V. Dua, and E. N. Pistikopoulos, "The explicit solution of model predictive control via multiparametric quadratic programming", in Proc. American Control Conference, Chicago, 2000, pp. 872-876. [5] T. A. Johansen, I. Petersen, and O. Slupphaug, "On explicit suboptimal LQR with state and input constraints", in Proc. IEEE Conf. Decision and Control, Sydney, 2000, pp. TuM05-6.

[6] M. Krstic, I. Kanellakopoulos, and P. Kokotovic, Nonlinear Adaptive Control Design, Wiley and Sons, 1995.

[7] E. D. Sontag, "A 'universal' construction of Artstein's theorem on nonlinear stabilization", Systems and Control Letters, vol. 13, pp. 117-123, 1989.

[8] J. Kalkkuhl, T. A. Johansen, J. Lüdemann, and A. Queda, "Nonlinear adaptive backstepping with estimator resetting using multiple observers", in Proc. Hybrid Systems, Computation and Control, Rome, 2001. 